\title{
Centralization vs. Decentralization in a Multi-Unit Organization: A Computational Model of a Retail Chain as a Multi-Agent Adaptive System*
}

\author{
Myong-Hun Chang \\ Department of Economics \\ Cleveland State University \\ Cleveland, $\mathrm{OH} 44115$ \\ 216-687-4523, -9206 (Fax) \\ m.chang@popmail.csuohio.edu
}

\author{
Joseph E. Harrington, Jr. \\ Department of Economics \\ The Johns Hopkins University \\ Baltimore, MD 21218 \\ 410-516-7615, -7600 (Fax) \\ joe.harrington@jhu.edu
}

May 1998, Revised September 1999

\begin{abstract}
A computational model of a retail chain is developed in which store managers continually search for better practices. Search takes place over a rugged landscape defined over the space of store practices. The main objective of this research is to determine how the amount of discretion given to store managers, as to how they run their stores, influences the rate of innovation at the store level. We find that greater decentralization enhances firm performance when stores' markets are sufficiently different, the horizon is sufficiently long, and markets are sufficiently stable.
\end{abstract}

\footnotetext{
*The comments and suggestions of Bruce Hamilton, Jon Harford, Scott Page, and Tim Van Zandt and participants at seminars at Rochester-Simon School of Business, Cleveland State, Georgetown, Universitat Pompeu Fabra, and Vanderbilt-Owen Graduate School of Management, and at the 1998 UBC Summer Industrial Organization Conference, 1999 Conference on Institutions: Complexity and Difficulty (Santa Fe Institute), 1999 Society for Computational Economics Conference, and Computational and Mathematical Organization Theory Workshop (INFORMS-1999 Meetings) are gratefully acknowledged.
} 


\section{Introduction}

What is the optimal degree of centralization within a firm? To what extent should lower level managers be given the authority to act independently of higher level management? In the case of a retail chain, this question takes the form of how much discretion corporate headquarters should give to store managers. Should a tightly-controlled set of operating

procedures be mandated or should store managers be given considerable leeway with respect to running their stores?

Historically, different organizations chose different answers to these questions. A recent contrast is provided by two discount department stores, Wal-Mart and Ames:

[Sam] Walton [founder of Wal-Mart] valued change, experimentation, and constant improvement. But he didn't just preach these values, he instituted concrete organizational mechanisms to stimulate change and improvement. Using a concept called "A Store Within a Store," Walton gave department managers the authority and freedom to run each department as if it were their own business.

Whereas Walton concentrated on creating an organization that would evolve and change on its own, Ames leaders dictated all changes from above and detailed in a book the precise steps a store manager should take, leaving no room for initiative. [Collins and Porras (1994), pp. 36-7]

While anecdotes are many, little is understood about how exactly organizational structure influences the performance of a retail chain. Is there one organizational structure that is best? Or does it depend on a chain's environment? If so, what are the pertinent features of the environment and how do they influence the relative performance of different organizational structures? The objective of this research is to provide some theoretical insight into these 
questions. The dimension of organizational performance that we focus on is the dynamic one alluded to in the description of Wal-Mart: the rate of improvement in store practices achieved through innovations and organizational learning.

Our model of a retail chain begins with the view that a store, at any given point in time, is characterized by its current operating practices or what Nelson and Winter (1982) would refer to as "routines." Given the set of embedded routines, an innovation is then viewed as a new way of running a store as represented by a change in routine. A store's performance (profit) depends on how its current set of operating practices matches up with what is desired by its consumers. New ideas represent a new point in store practice space and associated with that new point is a level of profit. Store profit, being defined over this store practice space, then forms a landscape over which the store manager can search for better practices through a standard hill-climbing rule. Markets are allowed to differ and, thus, the landscapes faced by different store managers can differ. Analogously, corporate headquarters searches over a landscape based upon chain profit. In that we presume it does not have detailed information about stores, headquarters is presumed incapable of selectively instituting practices but must instead mandate a practice chain-wide.

To briefly summarize our findings, a decentralized organizational structure outperforms a centralized one when stores' markets are sufficiently heterogeneous, consumers are not too sensitive to store practices, the horizon is sufficiently long, and the market environment is sufficiently stable. Otherwise, a superior profit path is achieved through centralization.

The conceptual framework employed in our research has two major components. First, 
innovation is viewed as an act of information creation that improves the organization's ability to satisfy the demands of its external market environment. Second, an organization is viewed as a collection of agents, each of whom is capable of generating new ideas. As such, the potential sources of innovative ideas are distributed among multiple agents, rather than concentrated at a single central authority. Together, these two views lead to the central thesis that the performance of a retail chain, as measured by its ability to respond to the external market environment, depends critically on the way it organizes the complex process of communicating and utilizing innovative ideas generated by multiple internal sources.

That there exists a crucial linkage between the optimal organizational structure and the external environmental contingencies has long been recognized by organizational theorists [Lawrence and Lorsch (1967), Mintzberg (1979), Burton and Obel (1995)]. This linkage has also been applied to understanding strategy implementation in diversified multi-unit business firms [Govindarajan $(1986,1988)$, Morrison and Roth (1993)]. Our work contributes to this literature by explicitly modelling the innovation process through which an organization responds to various aspects of its environment. Given our view of innovation as the process of information creation and communication, our work is also related to Radner (1993) and Van Zandt (1998) which look at how the allocation of computational tasks within a hierarchy affects the efficiency of organizational information processing. A related body of work addresses the issue of intra-organizational screening of new information, where agents at different organizational levels may have conflicting opinions about the value of some information [Sah and Stiglitz (1986), Chang and Harrington (1998)]. 
One of the major environmental contingencies in our model that affects the optimality of an organizational form is the heterogeneity in the markets that various stores serve. Given that the generation of information is distributed and the initial ownership of the new knowledge is private, the heterogeneity in external environments faced by the agents naturally introduces the potential for conflicting interests and, thus, the agency problem [Holmström (1979, 1982), Rotemberg and Saloner (1993), Baiman et al. (1995), Aghion and Tirole (1997), Chang and Harrington (1999)]. By assuming a fixed stream of new ideas, our work bypasses the issue of incentives in generating innovations and instead focuses on the design of organization for effective communication and utilization of available information. Nevertheless, it will be shown later that the relative effectiveness of a given organizational form is very much affected by the conflict of interests among agents.

We model innovation as the dynamic process by which a piece of information utilized in a given period further influences the creation, communication, and utilization of future innovation. This process is extensively researched in the body of literature commonly referred to as "organizational learning." ${ }^{1}$ Of particular relevance are organizational learning models that encompass boundedly rational agents experimenting with new ideas and making piecewise improvements [Cohen (1981), Levinthal and March (1981), Nelson and Winter (1982)]. While our research belongs to this literature that models organizational learning as adaptive search, the exact search mechanism we utilize is distinct. In our model, innovation is modelled as random search carried out in a finite fixed space of ideas. This particular approach is rooted in the concept of a fitness landscape, defined in a multidimensional 
space in which each attribute of an organization (retail chain or a store in our model) is represented by a dimension of the space and a final dimension indicating the performance (profitability) of the organization. An adaptation by an organization is then represented by movement on the landscape toward a location reflecting higher fitness value. In the context of population genetics, Kauffman (1993) demonstrated that the topography of the fitness landscape is determined by the degree of interdependence of the fitness contribution of the various attributes of an organism. Taking the Darwinian perspective from organizational ecology, Levinthal (1997) uses this connection in the context of organizational attributes to examine the effectiveness of organizational adaptation at the population level. Both Carley and Svoboda (1996) and Carley and Lee (1998) also utilize the search-over-rugged-landscape perspective in modelling organizational restructuring as adaptive search for better organizational design given a group of learning agents. ${ }^{2}$ Our work is an extension in this body of literature in that our model investigates the impact of internal structure on the effectiveness of organizational search on the rugged landscape, where the ruggedness arises from the complementarity among various dimensions of store operations.

\section{A Model of a Retail Chain}

A retail chain is composed of a corporate headquarters (HQ) and $M \geq 2$ stores. Each store is in a distinct market and has a set of $N$ practices such that store $i$ 's operation in any given period is fully described by a vector, $z^{i} \equiv\left(z_{1}^{i}, z_{2}^{i}, \ldots, z_{N}^{i}\right)$, where $z_{j}^{i}$ is store $i$ 's practice for the

$j$ th dimension of its operation and $z_{j}^{i} \in\{1, \ldots, R\}$ for all $i \in\{1, \ldots, M\}$ and $j \in\{1, \ldots, N\}$. 
Thus, there are $R$ feasible practices for each dimension and, at any point in time, a store is represented by a point in $\{1, \ldots, R\}^{N} .^{3}$

\subsection{Representation of a Store's Market}

All consumers in market $i$ shop at store $i, i \in\{1,2, \ldots, M\}$. Each consumer has an ideal set of store practices which is represented as an element of $\{(1, \ldots, 1), \ldots,(R, \ldots, R)\}$. Assuming consumer types are in this restricted set reflects complementarities in their preferences which could be due, for example, to different income levels. People with higher income may incur greater search costs so they would prefer everyday low prices with fewer sales (which avoids having to spend time searching for sales), fewer product lines and larger inventories (reducing the chances of being out-of-stock of a product and thus creating the need for another trip to the store), and more attentive though more aggressive sales personnel (which might speed up the time spent buying) as might be achieved by having sales personnel work on commission.

The consumer with an ideal set of store practices of $(w, \ldots, w)$ is denoted a type $w$ consumer. The utility to a type $w$ consumer from buying $x$ units at a price of $p$ from store $i$ is specified to be: $u\left(x ; p, w, z^{i}\right)=\left[\bar{L}-\sqrt{\sum_{j=1}^{N}\left(z_{j}^{i}-w\right)^{2}}\right]^{\gamma} \cdot x^{\beta}-p \cdot x$; where $\beta \in(0,1), \gamma \geq 1$, and $\bar{L}$ is chosen so that $\left[\bar{L}-\sqrt{\sum_{j=1}^{N}\left(z_{j}^{i}-w\right)^{2}}\right]>1$ for all $\left(w,\left(z_{1}^{i}, \ldots, z_{N}^{i}\right)\right)$. Letting $d \equiv$ $\sqrt{\sum_{j=1}^{N}\left(z_{j}^{i}-w\right)^{2}}$, it can be shown that $\bar{L}-d>1$ is sufficient for $\frac{\partial^{2} u}{\partial d \partial \gamma}<0$. Hence, an increase in $\gamma$ reflects a consumer's higher marginal dissatisfaction from actual store practices deviating from most preferred practices. Given the above utility function, a type $w$ consumer's optimal quantity decision is then: $x^{*}\left(p, w, z^{i}\right)=\left(\frac{\beta}{p}\right)^{\frac{1}{1-\beta}}\left[\bar{L}-\sqrt{\sum_{j=1}^{N}\left(z_{j}^{i}-w\right)^{2}}\right]^{\frac{\gamma}{1-\beta}}$. 
In market $i$, consumers are distributed according to a cdf $F_{i}:\{1, \ldots, R\} \rightarrow[0,1]$. Markets are homogeneous when $F_{1}=F_{2}=\cdots=F_{M}$. The computational model assumes the following specification regarding the distribution of consumer types. In a given market, 1000 consumers are distributed over the type space, $\{1,2, \ldots, 100\}$, according to a discrete density function which has positive density over 50 neighboring types, where the positive densities approximate a triangular density function.

\subsection{Representation of a Store and a Chain}

In any period, a store's type is represented by its current practices which is an element of $\{1, \ldots, R\}^{N}$. Given practices $z^{i} \equiv\left(z_{1}^{i}, \ldots, z_{N}^{i}\right)$, store $i$ 's current profit function is:

$$
(p-c) \int x^{*}\left(p, w, z^{i}\right) d F_{i}(w)=(p-c)\left(\frac{\beta}{p}\right)^{\frac{1}{1-\beta}} \int\left[\bar{L}-\sqrt{\sum_{j=1}^{N}\left(z_{j}^{i}-w\right)^{2}}\right]^{\frac{\gamma}{1-\beta}} d F_{i}(w)
$$

A store is assumed to optimally set its price. ${ }^{4}$ Using the profit function, the optimal

price is: $p^{*}=\frac{c}{\beta}$. The resulting demand from type $w$ consumers is then: $X_{i}^{*}\left(w, z^{i}\right)=$ $\left(\frac{\beta^{2}}{c}\right)^{\frac{1}{1-\beta}}\left[\bar{L}-\sqrt{\sum_{j=1}^{N}\left(z_{j}^{i}-w\right)^{2}}\right]^{\frac{\gamma}{1-\beta}}$. Store $i$ 's current profit is:

$\left[\left(\frac{c}{\beta}\right)-c\right] \int X_{i}^{*}\left(w, z^{i}\right) d F_{i}(w)=\left[\left(\frac{c}{\beta}\right)-c\right]\left(\frac{\beta^{2}}{c}\right)^{\frac{1}{1-\beta}} \int\left[\bar{L}-\sqrt{\sum_{j=1}^{N}\left(z_{j}^{i}-w\right)^{2}}\right]^{\frac{\gamma}{1-\beta}} d F_{i}(w)$

What is important for our analysis is that a store's profit is decreasing in the distance between its practices and those desired by its customers.

The profit for the chain is a simple sum of stores' profits:

$$
\left[\left(\frac{c}{\beta}\right)-c\right]\left(\frac{\beta^{2}}{c}\right)^{\frac{1}{1-\beta}} \sum_{i=1}^{M}\left[\int\left(\bar{L}-\sqrt{\sum_{j=1}^{N}\left(z_{j}^{i}-w\right)^{2}}\right)^{\frac{\gamma}{1-\beta}} d F_{i}(w)\right]
$$


The degree of centralization in a chain is measured by the number of dimensions that HQ controls. Let $\Omega \subseteq\{1, \ldots, N\}$ denote the set of dimensions controlled by HQ so that store managers control $\{1, \ldots, N\}-\Omega$. In the context of our model of innovation, control of a given dimension implies the authority to adopt those ideas that entail changes in that dimension. The exact specification is provided later.

\section{Structure of the Landscape}

A store's and a chain's landscapes can be thoroughly characterized by evaluating the profit functions (2.2) and (2.3), respectively, for all practices. Due to the computational constraint, however, we limit our evaluation in this section to only two-dimensional store practice space $(N=2)$. Given a triangular density with the peak consumer type at 50 and the parameter values of $R=100, M=2, c=10$, and $\beta=0.5$, let $z^{*}(\gamma) \equiv\left(z_{1}^{*}(\gamma), z_{2}^{*}(\gamma)\right)$ denote the local optimum of a landscape as a function of $\gamma$, where $z_{i}^{*}(\gamma)$ is the store's practice in the $i$ th

dimension at the local optimum. Table 1 provides the local optima for $\gamma \in\{1,3,5,7\}$. Note that complementarities in consumers' preferences lead to $z_{1}^{*}(\gamma)=z_{2}^{*}(\gamma)$.

Remark 1: An increase in $\gamma$ raises the number of local optima for a store. A store's landscape becomes more rugged as $\gamma$ rises.

Similarly, a chain's landscape, defined over the sum of stores' profits, tends to exhibit the same characteristics: an increase in $\gamma$ raises the number of local optima for a chain. ${ }^{5}$ 


\section{Modelling of Innovation}

While the results reported in this paper only have stores generating new ideas, let us describe the more general model that involves $\mathrm{HQ}$ also generating ideas. In each period, $\mathrm{HQ}$ and each store generates ideas. A typical idea has the following properties. An idea spans $K$ dimensions of the store, where $K \in\{1, \ldots, P\}$ and $P \in\{1, \ldots, N\}$ is a parameter. The $K$ dimensions are randomly selected from $\{1, \ldots, N\}$ without replacement. For each of those $K$ dimensions, there is a random draw from $\{1, \ldots R\}$. The degree of sophistication (or equivalently, the degree of complexity) in a given idea is measured by $P$, the maximum number of dimensions that an idea can encompass. For the remainder of the analysis we will fix $P=1$ so that all ideas are one-dimensional. ${ }^{6}$

To make the process of idea generation a bit more concrete, let us consider an example with $N=5, R=100$, and $P=1$. Suppose the current store practice is $(25,56,71,33,89)$ and an innovation involves changing the practice in the third dimension from 71 to 95 . The new store practice accommodating this change would be $(25,56,95,33,89)$. If this idea is implemented by another store currently employing $(11,29,54,49,65)$, its new store practice will be expressed as $(11,29,95,49,65)$.

In each period, the ideas generated by HQ and the stores are considered for adoption sequentially with the order being randomly determined. Recall that $\Omega \subseteq\{1, \ldots, N\}$ represents the dimensions controlled by HQ while store managers control $\{1, \ldots, N\}-\Omega$. A particular organizational form is then defined by $\Omega .^{7}$ Let $I_{h}^{t}$ and $I_{k}^{t}$ denote the dimension encompassed 
by the period $t$ idea of HQ and store $k$, respectively. Since ideas are one-dimensional then $I_{h}^{t}, I_{k}^{t} \in\{1, \ldots, N\}$

The adoption procedure in a given period is described by the following algorithm.

- Consider an idea generated by HQ. If $I_{h}^{t} \in \Omega$ then HQ has authority over the idea. If its adoption by all stores would raise chain profit then it is mandated. Otherwise, the idea is discarded. On the other hand, if $I_{h}^{t} \notin \Omega$ then HQ does not have authority over the idea. If at least $y$ stores, $y \in\{1, \ldots, M\}$, would benefit from the adoption of this idea then HQ passes the idea to all stores for their independent evaluation and adoption. Otherwise, the idea is discarded. The recommended idea is considered independently by each store's manager and adopted if it raises the store's profit. Otherwise, it is discarded by the store.

- Consider an idea generated by a store, $k$. If $I_{k}^{t} \in\{1, \ldots, N\}-\Omega$ then the manager of store $k$ has authority over the idea. He immediately adopts the idea if and only if it raises his store's profit. Otherwise, he discards the idea. If the idea is adopted by the store, it is then observed by HQ. ${ }^{8} \mathrm{HQ}$ passes it to all stores for potential adoption if and only if at least $y$ stores would benefit from its adoption. Otherwise, it is discarded at the HQ-level. The recommended idea is considered independently by each store's manager and adopted if it raises the store's profit. Otherwise, it is discarded by the store. Conversely, if $I_{k}^{t} \notin\{1, \ldots, N\}-\Omega$ then the manager of store $k$ does not have authority over the idea. He sends the idea up to HQ if its adoption would raise store 
$k$ 's profit. Otherwise, it is discarded at the store-level. The idea that is sent up to HQ is mandated if it raises the chain's profit. Otherwise, it is discarded at the HQ-level.

- " $y$ " is chosen to maximize long-run chain profit.

The last part requires some explanation. The task is to specify a reasonable rule for $\mathrm{HQ}$ to use in deciding whether to pass an idea to all stores. Since no particular rule obviously dominates, we consider a class of rules, defined by $y \in\{1, \ldots, M\}$, and choose that rule that maximizes chain performance. Notice that $y=1$ is equivalent to passing every idea to the stores so that $\mathrm{HQ}$ does not actively screen ideas. $y=M$ corresponds to passing an idea along only if it would be adopted by all stores so that uniform practices in the affected dimensions would prevail. ${ }^{9}$

Implicit in this adoption process is that a store manager and HQ know the associated profit from adopting a new idea. However, we do not suppose that they have global information about the landscape. Identifying a new point in store practice space represents an act of creativity. Having identified a new store practice, we suppose that a store (HQ) can experiment with it so as to reveal the associated store (chain) profit from its adoption. Rather than model this process of experimentation, which would further complicate the model, we implicitly assume it is done instantly and costlessly. To motivate the use of a $y$ rule, we imagine that HQ has an idea of the mix of different market types served by their chain but does not know which store serves which market. ${ }^{10}$

Note that in our model the internal diffusion of ideas is facilitated solely by HQ. While 
we recognize the possibility of direct inter-store learning, it is not clear that this separate chain of communication will have any qualitative impact on our analysis though the matter is worthy of further consideration. ${ }^{11}$

\section{Stable Markets and Convergence to Local Optima}

\subsection{Simulation Design ${ }^{12}$}

Two extreme organizational forms are considered: full decentralization (store managers control all dimensions and $y$ is chosen to maximize long-run chain profit) and full centralization (HQ controls all dimensions). ${ }^{13}$ For each set of parameter values, the computational experiment consists of 500 replications of the innovation procedure. Each replication involves a randomly drawn vector of initial store practices (which are assumed to be identical for the stores) and a sequence of $T M$ new practices, one for each of the $M$ stores in each of $T$ periods. We let $T \in\{500,1000,1500\}$. Two classes of output data were collected for each set of parameter values. First, the ex ante optimal organizational form based on average chain profit over the horizon averaged over the 500 replications. Second, the frequency with which an organizational form is the ex post optimum (out of the 500 replications) in terms of average chain profit over the horizon.

For tractability, we assume that $M$ markets can be classified into two separate types on the basis of consumer distributions. More specifically, there are two cumulative distribution functions, $F_{I}(w)$ and $F_{I I}(w)$, such that $F_{i}(w)=F_{I}(w)$ for $i=1, \ldots, \frac{M}{2}$ and $F_{i}(w)=F_{I I}(w)$ for $i=\frac{M}{2}+1, \ldots, M$. In any given market of type $I(I I), 1000$ consumers are distributed over the type space, $\{1,2, \ldots, 100\}$, according to a discrete density function $f_{I}\left(f_{I I}\right)$ generated 
from $F_{I}\left(F_{I I}\right) \cdot f_{I}\left(f_{I I}\right)$ has a triangular density over 50 neighboring types. Letting $\bar{w}_{I}$ and $\bar{w}_{I I}$ denote the peak (dominant) consumer types in markets of type $I$ and $I I$, respectively, it is assumed that $\bar{w}_{I}=50-\alpha$ and $\bar{w}_{I I}=50+\alpha$, where $\alpha$ is the degree of inter-market heterogeneity. Results are presented only for $M=2$ though have been found to be qualitatively robust to when $M=4$ and $M=6$.

For the simulations, the following parameter values were assumed: $\beta=0.5, c=10$ for $\gamma \in\{1,3,5\}$ and $c=10,000$ for $\gamma=7,{ }^{14} R=100, N=10, K=P=1, \Omega \in\{0,10\}, M=2$, $\alpha \in\{0,1,2,3,4,5\}, \gamma \in\{1,3,5,7\}$,and $S=500$ (\# of replications). We further assume that HQ generates no ideas while each store manager generates one idea per period. All stores are endowed with an identical set of practices that is randomly selected from $\{1, \ldots, 100\}^{10}$.

\subsection{Results}

The numerical output is presented in Tables 2 and 3 . Table 2 reports the organizational structure that generated higher average chain profit over the first $T$ periods (with that average profit being averaged over the 500 replications). Table 3 reports the frequency, out of the 500 replications, with which a given organizational structure yielded higher average chain profit over the first $T$ periods.

Property 1: Centralization is more likely to outperform decentralization when markets are sufficiently similar ( $\alpha$ low), while decentralization is more likely to outperform centralization when markets are sufficiently different ( $\alpha$ high). 
Examining Table 2, decentralization outperforms centralization in terms of average performance (averaged over the 500 replications) when $\alpha>2$. Centralization outperforms when $\alpha=0, \alpha=1$ (and $\gamma=3,5,7)$, and $\alpha=2(\gamma=5,7$ and $T=500) .{ }^{15}$ Turning to Table 3 and considering, for example, $(\gamma, T)=(5,500)$, we find that average profit (over the horizon) for centralization exceeded that for decentralization in 268 of the 500 runs when $\alpha=1$ (compared to 225 runs in which decentralization outperformed centralization), 222 runs when $\alpha=2$ (compare to 278), and 152 runs when $\alpha=3$ (compared to 348 ).

Since centralization imposes uniformity of practices, it is not surprising that decentralization outperforms when stores' markets are sufficiently different. In that situation, it is preferable to allow each store manager to tailor practices to his own unique market. Requiring common practices, as occurs under centralization, would involve, for at least one market, establishing practices that are quite inadequate for pleasing the market's consumers.

What is more intriguing is that centralization is preferred when markets are not too different. The benefits of decentralization are clear as it allows each store to tailor its practices to its market. What we believe to be the detrimental aspect is that as stores come to target different consumers the extent to which they learn from each other's practices diminishes. For example, if store 1 comes to target consumer 45 (presumably because the peak of the consumer distribution in store 1's market is close to 45) and store 2 comes to target consumer 53 then a practice implemented by store 1 - which might make some dimension have a value of, say, 46 - is unlikely to raise store profit if adopted by store 2 . In contrast, if both stores target consumer 49 then each store can learn from one another and 
this can lead to higher chain profit even if neither store is achieving a global optimum for its market. In short, there is a significant short-run advantage to the uniformity induced under centralization in that it promotes spillover of ideas across stores.

In order to substantiate this conjectured explanation of Property 1, let us begin by examining a single run. For a chain with two stores, there are two ideas to be evaluated in any period so that, over an horizon of 1500 periods, there is a sequence of 3000 ideas. To each idea we assign a number from $\{0,1,2\}$ which is equal to the number of stores that would benefit from that idea (that is, its profit would rise) given the store practices for that period. What we need to show to validate our conjecture is that there is a greater number of ideas benefitting both stores under centralization than under decentralization. For $\alpha=1$, Figure 1 shows the resulting series under decentralization while Figure 2 shows it under centralization. First note that the pattern is roughly the same during the first 300 periods or so in that many ideas are beneficial to both stores under either structure. Thereafter the patterns diverge as, under decentralization, the frequency of ideas that would benefit both stores decreases dramatically. Most of the ideas generated from then on tend to benefit only one store. In contrast, the presence of mutually beneficial ideas persists under centralization so that what one store discovers and adopts is often desirable to the other store.

The above evidence was for a single simulation run. We next performed a large number of runs and constructed a measure of the likelihood that an idea in a given period benefits both stores. Five hundred replications of the previous simulation were carried out, each time using a fresh set of initial practices and sequence of 3,000 ideas. For each replication, the 
number of stores benefitted by an idea was calculated for each idea. Upon completing the 500 runs, we computed for each organizational structure and for each element of the 3,000 length sequence, the frequency of the cases that benefitted both stores as a fraction of the 500 runs. Figure 3 captures the differential proportion under centralization and decentralization for $(\alpha, \gamma)=(1,3)$; that is, the proportion of runs in which a given idea benefitted both stores under centralization minus the same measure under decentralization. It shows that a centralized organization has a greater likelihood of generating ideas that benefit both stores. $^{16}$

Property 2: Centralization is more likely to outperform over short horizons $(T=500)$ while decentralization is more likely to outperform over long horizons $(T=1500)$.

An important factor in this result is that the global optimum under decentralization has higher chain profit than the global optimum under centralization. A decentralized structure allows for the possibility that each store achieves its global optimum by exactly tailoring its practices to what is desired by its consumers. Centralization rules out that possibility by virtue of mandating common practices. As achieving a global optimum is more likely with a longer horizon, decentralization tends to perform relatively better as the horizon increases.

Property 3: Centralization is more likely to outperform when consumers are sufficiently sensitive to store practices ( $\gamma$ high), while decentralization is more likely to outperform when consumers are sufficiently insensitive to store practices $(\gamma$ low). 
Recalling Remark 1, Property 3 implies that the relative effectiveness of centralization rises with the ruggedness of the landscapes faced by the stores and the chain. To understand this result, compare $\gamma=1$ and $\gamma=3$ when $\alpha=2$. The set of local optima is shown in Table 4. Consider $(\alpha, \gamma)=(2,1)$. If store 1 receives an idea converting some dimension to, say, 48 , this idea is unlikely to generate beneficial inter-store spillovers under either organizational form. While it is apt to be valuable to store 1 (as it has a local optimum at 48), an idea with value 48 is neither part of a local optimum for store 2 - so that under decentralization it would probably not be adopted by store 2 - nor part of a local optimum for the chain so that under centralization, HQ would probably not mandate the idea. Now consider that same idea when $\gamma=3$. Under decentralization, it probably will not result in inter-store learning as 48 still fails to be part of a local optimum for store 2. However, it is now part of a local optimum for the chain so that the idea, when passed by store 1 to HQ, is likely to be mandated under centralization. More generally, as $\gamma$ increases, the set of local optima expands so that there is more overlap between the local optima of stores and between the local optima of the chain and a store. However, it seems that the overlap between the chain and stores expands faster. In the example above, the set of overlap between the chain and store $1(2)$ expands from $\{49\}$ to $\{48,49,50\}(\{50,51,52\})$ while the set of overlap between the optima of the stores goes from the empty set to $\{50\}$. Hence, it becomes more likely that a store will pass an idea up to HQ that HQ finds to enhance chain profit. What this means is that the opportunity for inter-store spillovers is expanding at a faster rate under centralization relative to decentralization, as $\gamma$ is increased. While the increased ruggedness 
of stores' landscapes enhances inter-store learning under decentralization, the opportunities accelerate faster under centralization. ${ }^{17}$

The basic force underlying the results of this section is the following trade-off associated with a more decentralized structure. Moving authority down the hierarchy allows store managers to modify their operational routines over time so that each store's operation is reasonably well-adapted to its local market environment. The downside to this uncoordinated process of improvement is that it may lead to an eventual divergence in practices across stores. As stores migrate to different parts of the landscape, a new practice uncovered and adopted at one store will be incompatible with the current practices of other stores in the chain. In essence, stores gradually come to target distinct consumers and this limits the extent of inter-store learning. This has the effect of slowing down the rate of innovation as stores end up searching independently. The upside is that the practices that they do adopt, though fewer in number, are better suited to their environment. In contrast, a centralized structure enhances inter-store learning by mandating common practices and keeping stores at the same point on the landscape. With these two countervailing effects, we find that a decentralized structure outperforms only when markets are sufficiently heterogeneous, the horizon is sufficiently long, and consumers are sufficiently insensitive to store practices.

\section{Fluctuating Markets and Perpetual Innovation}

The previous analysis showed that centralization can outperform decentralization but only in the short-run. The market environment was specified to be unchanging so that the chain 
and stores were searching a fixed landscape. Now let us enrich this model by allowing the landscape to change due to movements in consumer preferences. How does a continually changing environment alter the relative performance of these organizational structures?

Given the same triangular density for consumer types as assumed in stable markets, let $x_{i}^{t}$ denote the peak type in market $i$ in period $t$ so that positive densities of consumer types range from $x_{i}^{t}-25$ to $x_{i}^{t}+25$. Our focus is on market fluctuations in which the distribution of consumers shifts over time in a stochastic manner. Allowing for the possibility that the changes in consumer tastes may be correlated across markets, we introduce a parameter $\rho$ which is the probability that the market shifts in any given period are perfectly correlated. With the probability $(1-\rho)$, we assume that the market shifts are entirely independent of one another.

When $\rho<1.0$, there is apt to be a built-in bias for decentralization induced by the increasing degree of cross-market heterogeneity over time. To control for this artificial bias, we restrict the movement of the peak consumer types to a fixed interval between $50-A$ and $50+A$. The simulation results reported here assume $A=8$, though the qualitative results were found robust to $A=2$ and $A=4$.

Let $x_{i}^{0}$ be the peak type of the initial consumer distribution in market $i$ at $t=0$, where $x_{i}^{0}$ is randomly drawn from $\{50-A, \ldots, 50+A\}, i=1,2$. In each period from $t=1$ and on, the triangular densities in either or both markets shift by one unit with probability $d$ and remain unchanged with probability $1-d$. These changes are assumed to be perfectly correlated with probability $\rho$ and independent with probability $(1-\rho)$. The exact market 
shift dynamics follow the algorithm specified below:

- With probability $\rho$, the market shifts are perfectly correlated:

$$
\begin{aligned}
& \text { If } 50-A<x_{1}^{t}<50+A \text { and } 50-A<x_{2}^{t}<50+A, \\
& \text { then }\left(x_{1}^{t+1}, x_{2}^{t+1}\right)= \begin{cases}\left(x_{1}^{t}+1, x_{2}^{t}+1\right) & \text { with probability } d / 2, \\
\left(x_{1}^{t}, x_{2}^{t}\right) & \text { with probability } 1-d, \\
\left(x_{1}^{t}-1, x_{2}^{t}-1\right) & \text { with probability } d / 2 .\end{cases} \\
& \text { If } x_{1}^{t}=50-A \text { and/or } x_{2}^{t}=50-A, \\
& \text { then }\left(x_{1}^{t+1}, x_{2}^{t+1}\right)= \begin{cases}\left(x_{1}^{t}, x_{2}^{t}\right) & \text { with probability } 1-d, \\
\left(x_{1}^{t}+1, x_{2}^{t}+1\right) & \text { with probability } d .\end{cases} \\
& \text { If } x_{1}^{t}=50+A \text { and/or } x_{2}^{t}=50+A, \\
& \text { then }\left(x_{1}^{t+1}, x_{2}^{t+1}\right)= \begin{cases}\left(x_{1}^{t}, x_{2}^{t}\right) & \text { with probability } 1-d, \\
\left(x_{1}^{t}-1, x_{2}^{t}-1\right) & \text { with probability } d .\end{cases}
\end{aligned}
$$

- With probability $1-\rho$, the market shifts are independent:

$$
\begin{aligned}
& \text { If } 50-A<x_{i}^{t}<50+A, \quad \text { then } x_{i}^{t+1}= \begin{cases}x_{i}^{t}+1 & \text { with probability } d / 2, \\
x_{i}^{t} & \text { with probability } 1-d, \\
x_{i}^{t}-1 & \text { with probability } d / 2 . \\
x_{i}^{t} & \text { with probability } 1-d, \\
x_{i}^{t}+1 & \text { with probability } d . \\
x_{i}^{t} & \text { with probability } 1-d, \\
x_{i}^{t}-1 & \text { with probability } d .\end{cases} \\
& \text { If } x_{i}^{t}=50-A,
\end{aligned}
$$

for $i=1$ and 2 .

Using the above rule of market dynamics, the search process is simulated over 1500 periods. The structure is otherwise identical to that specified in the preceding section and indeed that model is the special case of $d=0$ with $\left(x_{1}^{0}, x_{2}^{0}\right)$ being fixed and symmetric around type 50 .

When market fluctuation is allowed, two technical problems arise in comparing the simple profit path of the chain under different organizational forms. First, there is typically an initial transition to the point where profit is fluctuating around some steady-state mean. Second, market dynamics can cause considerable randomness in the profit path. Figure 4 obtained from a single simulation with $\rho=1.0$ and $d=1.0$ captures a typical profit path 
exhibiting these properties in the presence of severe market volatility. To surmount these two obstacles to estimating long-run profit, we use Welch's procedure to average the output processes generated from multiple replications. ${ }^{18}$ Figure 5 plots a moving average profit path as described in Welch's procedure for $\rho=1.0$ and $d=1.0$, where the value of the profit at $t=1000$, for instance, is an average of the profits from $t=500$ to $t=1500$. It seems quite clear from Figure 5 that the moving average at $t=1000$ is on the steady-state path. Based on this evidence, we put forth the following performance measure.

Definition 6.1. The performance criterion in the presence of market fluctuations is singleperiod profit averaged (across 500 replications) from $t=500$ to $t=1500$.

Using the above performance criterion, the ex ante optimal organizational forms (as specified previously for stable markets) are reported in Table 5 for different pairs of $(\rho, d)$ for $\gamma=3$ and $\gamma=5$. The following property appears to be present when the market environment is subject to random fluctuations.

Property 4: Centralization is more likely to outperform decentralization when market fluctuations are sufficiently large ( $d$ is close to 1 ) while decentralization is more likely to outperform when market fluctuations are sufficiently small ( $d$ is close to 0$)$.

Property 4 is quite consistent with the findings of the previous section. There we found that centralization performs better over shorter horizons which suggests that centralization results in stores learning at a faster rate when their practices are farther away from an optimum. In that a higher value of $d$ means more change in the landscape, it results in 
the stores being pushed farther away from an optimum. With periodic market shocks of that sort, stores may continually find themselves with highly sub-optimal practices. Hence, it is not surprising that greater market volatility enhances the relative performance of a centralized structure. We then find that changes in stores' environments may result in a centralized structure being preferred even in the long-run.

As seen in Table 5, Property 4 is robust to varying degrees of correlation among market fluctuations. Furthermore, we note the following property with respect to $\rho$.

Property 5: Centralization is more likely to outperform decentralization when market fluctuations are less correlated ( $\rho$ close to 0 ) while decentralization is more likely to outperform when market fluctuations are more strongly correlated ( $\rho$ close to 1 ).

Shocks to consumer preferences that are common across all markets then favor decentralized organization, while market-specific shocks tend to favor centralized organization. Unfortunately, we are unable to come up with an explanation for this phenomenon.

\section{Conclusion}

In this paper, we constructed a computational model of a retail chain in which each store manager continually searches for better practices through innovation. The search was carried out over a rugged landscape defined over the space of store practices with profit being determined by the interaction of a store's current practices and the distribution of consumer preference in its market. Given a fixed process by which new ideas are generated, organizational structure influences the process by which new ideas become new practices. The 
relative performance of centralized and decentralized organizational structures were compared in terms of profit generated over the short-run and long-run.

When the market environment is fixed over time, centralization was found to outperform decentralization in the short run when markets are relatively homogeneous and consumers are sufficiently sensitive to store practices. The primary force at work is that the centralized retail chain induces uniformity in store practices. By keeping stores at the same point on the landscape, this promotes inter-store learning; what is uncovered and passed along by one store is apt to be of value to other stores. Centralization then promotes effective sharing of ideas and this results in a rapid convergence to an optimum. The superior performance of centralization for some market environments is restricted, however, to the short run because the global optimum under centralization is inherently inferior to that under decentralization as a result of restricting stores to having the same practices. Under decentralization, each store can, in the long-run, more effectively tailor its practices to its own market and thereby this organizational form eventually outperforms a more centralized organization.

When the analysis is extended to accommodate the possibility of a changing market environment, the short-run superiority of a centralized retail chain can be extended to the long run. With the market environment continually changing, the spillovers between stores that is promoted under centralization becomes a dominant force. Quite contrary to the usual claim that volatility in markets requires a more flexible decentralized organizational form, we find that it is the centralized organization with coordinated search that is more effective in responding to a new environment. 


\section{Endnotes}

${ }^{1}$ Recent works representative of this category can be found in Cohen and Sproull (1996).

${ }^{2}$ The same perspective is used by Kauffman et al. (1998) and Auerswald et al. (1998) in modeling technological innovation. Kollman et al. (1998) offer another context for its application: they examine how institutional structure influences the efficacy of search for better solutions within the context of federal systems using states as policy laboratories.

${ }^{3}$ These practices represent all those elements that influence the appeal of this store to consumers. It can include the types of products carried (Target has more fashion-oriented clothing than Wal-Mart; Discount Store News, 4/1/96), the number of products carried (Kohl's product line is narrow but deep; Discount Store News, 4/1/96), and compensation schemes (Sears reduced performance-based incentives for store employees during the 1970s and restored them by 1997; Harvard Business School Case Study, N9-898-007).

${ }^{4}$ More explicitly, one could incorporate the learning procedure into the model so that the stores may experiment with many different prices in any given period for a chosen set of practices. Our belief is that experimentations on price can be carried out much more often than those on store practices. Moreover, that the optimal price is independent of store practices favors the presumption that price experimentation in the beginning of the horizon reveals the optimum rather quickly and, henceforth, no further price search is necessary.

${ }^{5}$ Profit values at the local optima reveal that both a store's and the chain's landscapes form a massif. There is a central global optimum surrounded by inferior local optima. The value of a local optimum monotonically declines in its distance from the central peak. 
${ }^{6}$ Additional simulations for $P \in\{3,5,10\}$ show that all of our qualitative results are robust.

${ }^{7}$ Actually, $\Omega^{\prime}$ and $\Omega^{\prime \prime}$ are equivalent if $\left|\Omega^{\prime}\right|=\left|\Omega^{\prime \prime}\right|$ so that it would be more exact to define an organizational form by $|\Omega|$.

8 "... the real job of a district manager [at J.C. Penney] is to bring information and guidance from the central office to the store manager and to bring to the central office information they gather from the stores; but, more than that, to pollenize all stores in their territories with whatever useful information they gather while visiting them." [Beasley (1948), p. 235]

${ }^{9}$ For each organizational form, the profit paths over 1500 periods are generated for all $y \in\{1, \ldots, M\}$ and the average profits are then compared to identify the optimal $y^{*}$ for the given organizational form. The profit path corresponding to $y^{*}$ is then used for the purpose of comparing between the organizational forms. A more general approach would be to define an organizational form by the pair $(|\Omega|, y)$ and contrast the performance of all possible pairs. Presenting so much information would be overwhelming so we instead just compare $\left(|\Omega|, y^{*}(|\Omega|)\right)$ where $y^{*}(|\Omega|) \in \arg \max V(|\Omega|, y)$ and $V(\cdot)$ is the chain's payoff.

${ }^{10}$ With large changes in store practices, in practice a chain will often if not always institute it in a few stores as a form of experimentation. If the experiment is successful then new practice will be adopted chain-wide. While experimentation is then a relevant feature of this process, we chose not to overload the model with too many features at once.

${ }^{11}$ See Darr, Argote, and Epple (1995) for a discussion of the impact that such direct 
learning has on organizational performance within the context of franchised stores.

${ }^{12}$ The simulation programs were written in ANSI standard C and compiled and run on VAX and ALPHA systems. The source code is available upon request from Myong-Hun Chang.

${ }^{13}$ Initial runs considered all possible organizational forms which means all values of $|\Omega|$ from $\{0,1, \ldots, N\}$. However, we found that the optimal structure was almost always either full centralization, $|\Omega|=N$, or full decentralization, $|\Omega|=0$. To save on computational time, we focused our attention on those two structures.

${ }^{14} c$ was raised to 10,000 for $\gamma=7$ so as to deflate the magnitude of profits. It only affects the absolute magnitudes without having any qualitative impact on our comparison of organizational forms.

${ }^{15}$ The optimal value of $y$ under decentralization tended to be 1 so that it is optimal to couple a decentralized structure with indiscriminate transfer of ideas by HQ.

${ }^{16}$ Additional simulations confirmed these results for $(\alpha, \gamma) \in\{(1,1),(3,1),(3,3)\}$.

${ }^{17}$ That inter-store learning under decentralization increases with landscape ruggedness is directly evidenced by the average distance between stores falling as $\gamma$ rises. When $\alpha=1$, average store distance falls from 4.43 to 3.61 to 3.01 as $\gamma$ rises from 1 to 3 to 5 . When $\alpha=3$, average store distance falls from 10.68 to 8.94 to 7.70 as $\gamma$ rises from 1 to 3 to 5 . With more local optima, it is more likely that stores will end up targetting the same optimum.

${ }^{18}$ Detailed discussions of this procedure are in Law and Kelton (1991) and Welch (1983). 


\section{References}

[1] Aghion, P. and J. Tirole (1997), "Formal and Real Authority in Organizations," Journal of Political Economy, 105, 1-29.

[2] Auerswald, P., S. Kauffman, J. Lobo, and K. Shell (1998), "The Production Recipes Approach to Modeling Technological Innovation: An Application to Learning By Doing," Cornell University, CAE Working Paper 98-10.

[3] Baiman, S., D. Larcker, and M. V. Rajan (1995), "Organizational Design for Business Units," Journal of Accounting Research, 33 (2), 205-229.

[4] Beasley, N. (1948), Main Street Merchant: The Story of the J. C. Penney Company. New York: Whittlesey House.

[5] Burton, R. and B. Obel (1995), Strategic Organizational Design and Diagnosis, Boston, Kluwer Academic Press.

[6] Carley, K. M. and D. M. Svoboda (1996), "Modeling Organizational Adaptation as a Simulated Annealing Process," Sociological Methods 83 Research, 25 (1), 138-168.

[7] Carley, K. M. and J.-S. Lee (1998), "Dynamic Organizations: Organizational Adaptation in a Changing Environment," Advances in Strategic Management, 15, 269-297.

[8] Chang, M.-H. and J. E. Harrington, Jr. (1998), "Organizational Structure and Firm Innovation in a Retail Chain," Computational and Mathematical Organization Theory, $3(4), 267-288$. 
[9] Chang, M.-H. and J. E. Harrington, Jr. (1999), "Decentralized Business Strategies in a Multi-Unit Firm," Unpublished Manuscript.

[10] Cohen, M. D. (1981), "The Power of Parallel Thinking," Journal of Economic Behavior and Organization, 2, 285-306.

[11] Cohen, M. D. and L. S. Sproull, eds. (1996), Organizational Learning, Thousand Oaks, CA: Sage Publications.

[12] Collins, J. C. and J. I. Porras (1994), Built to Last: Successful Habits of Visionary Companies, New York: HarperBusiness.

[13] Darr, E. D., L. Argote, and D. Epple (1995), "The Acquisition, Transfer, and Depreciation of Knowledge in Service Organizations: Productivity in Franchises," Management Science, 41 (11), 1750-1762.

[14] Govindarajan, V. (1986), "Decentralization, Strategy, and Effectiveness of Strategic Business Units in Multibusiness Organizations," Academy of Management Review, 11 (4), 844-856.

[15] Govindarajan, V. (1988), "A Contingency Approach to Strategy Implementation at the Business-Unit Level: Integrating Administrative Mechanisms with Strategy," Academy of Management Journal, 31 (4), 828-853.

[16] Holmström, B. (1979), "Moral Hazard and Observability," Bell Journal of Economics, 10, 74-91. 
[17] Holmström, B. (1982), "Moral Hazard in Teams," Bell Journal of Economics, 13, 32440.

[18] Kauffman, S. (1993), The Origins of Order, New York: Oxford University Press.

[19] Kauffman, S., J. Lobo, and W. G. Macready (1998), "Optimal Search on a Technology Landscape," Santa Fe Institute, 98-10-091.

[20] Kollman, K., J. H. Miller, and S. E. Page, "Searching for Solutions in a Federal System," University of Iowa, photocopy, April 1998.

[21] Law, A. M. and W. D. Kelton (1991), Simulation Modeling and Analysis, Second Edition, McGraw-Hill, Inc.

[22] Lawrence, P. R. and J. W. Lorsch (1967), Organization and Environment: Managing Differentiation and Integration, Boston, Graduate School of Business Administration, Harvard University.

[23] Levinthal, D. A. (1997), "Adaptation on Rugged Landscapes," Management Science, $43(7), 934-950$.

[24] Levinthal, D. and J. G. March (1981), "A Model of Adaptive Organizational Search," Journal of Economic Behavior and Organization, 2, 307-333.

[25] Mintzberg, H. (1979), The Structuring of Organizations, Englewood Cliffs, New Jersey, Prentice Hall. 
[26] Morrison, A. and K. Roth (1993), "Relating Porter's Configuration/Coordination Framework to Competitive Strategy and Structural Mechanisms: Analysis and Implications," Journal of Management, 19 (4), 797-818.

[27] Nelson, R. R. and S. G. Winter (1982), An Evolutionary Theory of Economic Change, Cambridge, MA.: The Belknap Press of Harvard University Press.

[28] Radner, R. (1993), "The Organization of Decentralized Information Processing," Econometrica, 61, 1109-1146.

[29] Rotemberg, J. J. and G. Saloner (1993), "Leadership Style and Incentives," Management Science, 39, 1299-1318.

[30] Sah, R. K. and J. E. Stiglitz (1986), "The Architecture of Economic Systems: Hierarchies and Polyarchies," American Economic Review, 76, 716-727.

[31] Van Zandt, T. (1998), "Real-Time Hierarchical Resource Allocation," Northwestern University, Center for Mathematical Studies in Economics and Management Science, Discussion Paper No. 1231.

[32] Welch, P. D. (1983), "The Statistical Analysis of Simulation Results," in The Computer Performance Modeling Handbook, S. S. Lavenberg, ed., New York: Academic Press. 
Table 1: Local Optima for a Store with $N=2$

\begin{tabular}{|c|c|c|c|c|c|c|c|c|}
\hline \multicolumn{9}{|c|}{ Set of Local Optima for a Store } \\
\hline$\gamma$ & & & & & $z^{*}(\gamma)$ & & & \\
\hline 1 & & & & $(49,49)$ & $(50,50)$ & $(51,51)$ & & \\
\hline 3 & & & $(48,48)$ & $(49,49)$ & $(50,50)$ & $(51,51)$ & $(52,52)$ & \\
\hline 5 & & $(47,47)$ & $(48,48)$ & $(49,49)$ & $(50,50)$ & $(51,51)$ & $(52,52)$ & $(53,53)$ \\
\hline 7 & $(46,46)$ & $(47,47)$ & $(48,48)$ & $(49,49)$ & $(50,50)$ & $(51,51)$ & $(52,52)$ & $(53,53)$ \\
\hline
\end{tabular}

Table 2: Ex Ante Optimum (Static Markets)

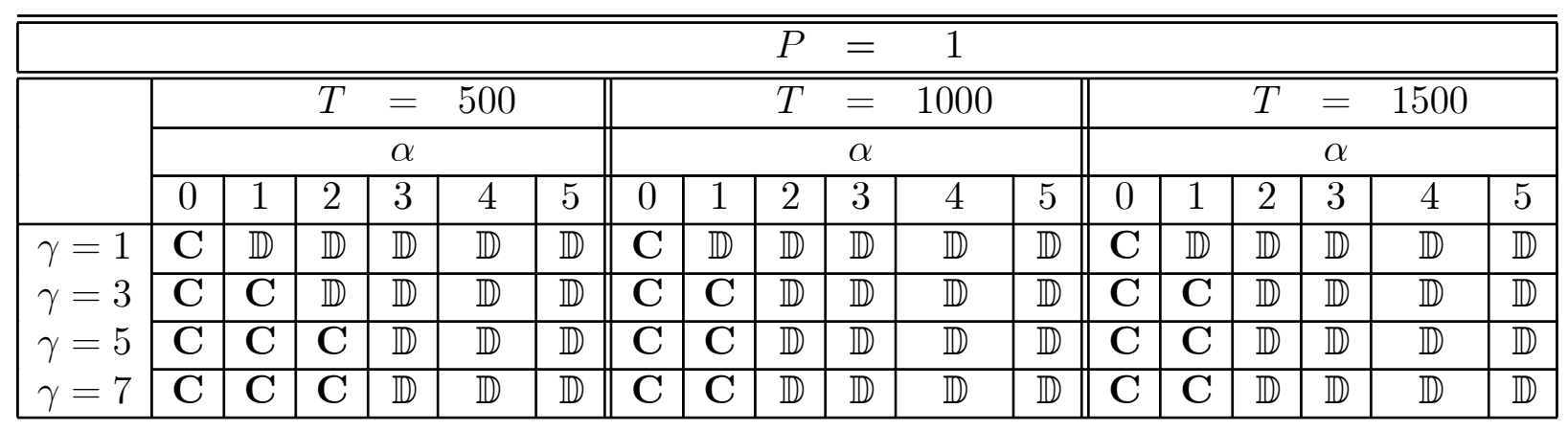




\section{Table 3: Frequency of Ex Post Optimality}

500 replications

D: HQ control $=0$ preferred.

C: HQ control $=10$ preferred.

$\mathrm{N}$ : Indifference.

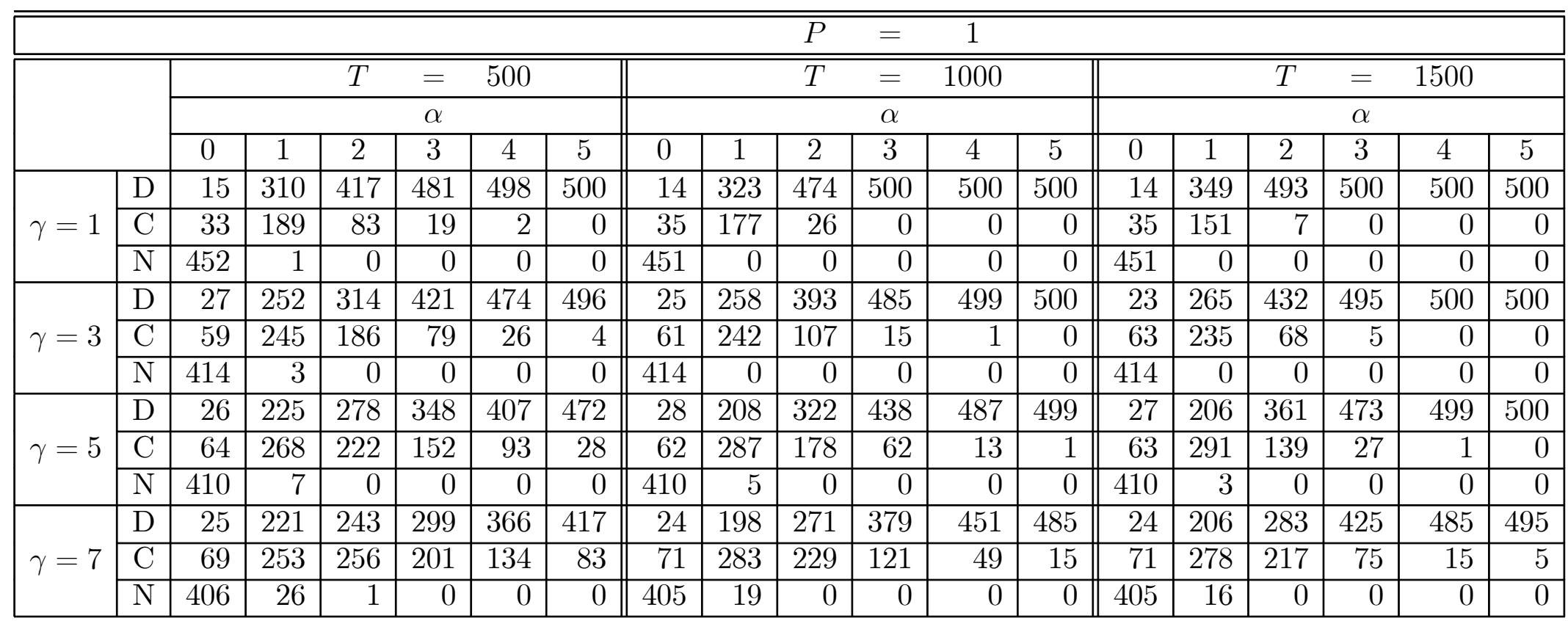


Table 4: Local Optima for Stores and Chain

\begin{tabular}{|c||c|c|}
\multicolumn{4}{c}{ Set of Local Optima } \\
\hline & $(\alpha, \gamma)=(2,1)$ & $(\alpha, \gamma)=(2,3)$ \\
\hline \hline Store 1 & $\{47,48,49\}$ & $\{46,47,48,49,50\}$ \\
\hline Store 2 & $\{51,52,53\}$ & $\{50,51,52,53,54\}$ \\
\hline Chain & $\{49,50,51\}$ & $\{48,49,50,51,52\}$ \\
\hline
\end{tabular}

Table 5: Ex Ante Optimum (Fluctuating Markets)

\begin{tabular}{|c|c|c|c|c|c|c|c|c|c|c|c|}
\hline & & & $\gamma$ & $=$ & 3 & ; & $A$ & $=$ & \multicolumn{3}{|l|}{8} \\
\hline & \multicolumn{11}{|c|}{$d$} \\
\hline & 0.0 & 0.1 & 0.2 & 0.3 & 0.4 & 0.5 & 0.6 & 0.7 & 0.8 & 0.9 & 1.0 \\
\hline$\rho=1.0$ & $\mathbb{D}$ & $\mathbb{D}$ & $\mathbb{D}$ & $\mathbb{D}$ & $\mathbb{D}$ & $\mathbb{D}$ & $\mathbb{D}$ & $\mathbb{D}$ & $\mathbb{D}$ & $\mathbb{D}$ & $\mathbb{D}$ \\
\hline$\rho=0.7$ & $\mathbb{D}$ & $\mathbb{D}$ & $\mathbb{D}$ & $\mathbb{D}$ & $\mathbb{D}$ & $\mathbb{D}$ & $\mathrm{C}$ & $\mathrm{C}$ & $\mathrm{C}$ & $\mathrm{C}$ & $\mathrm{C}$ \\
\hline$\rho=0.3$ & $\mathbb{D}$ & $\mathbb{D}$ & $\mathbb{D}$ & $\mathrm{C}$ & $\mathrm{C}$ & $\mathrm{C}$ & $\mathrm{C}$ & $\mathrm{C}$ & $\mathrm{C}$ & $\mathrm{C}$ & $\mathrm{C}$ \\
\hline$\rho=0.0$ & $\mathbb{D}$ & $\mathbb{D}$ & $\mathrm{C}$ & $\mathrm{C}$ & $\mathrm{C}$ & $\mathbf{C}$ & $\mathrm{C}$ & $\mathrm{C}$ & $\mathrm{C}$ & $\mathrm{C}$ & $\mathrm{C}$ \\
\hline
\end{tabular}

\begin{tabular}{|c|c|c|c|c|c|c|c|c|c|c|c|}
\hline & & & $\gamma$ & $=$ & 5 & ; & $A$ & $=$ & \multicolumn{3}{|l|}{8} \\
\hline & \multicolumn{11}{|c|}{$d$} \\
\hline & 0.0 & 0.1 & 0.2 & 0.3 & 0.4 & 0.5 & 0.6 & 0.7 & 0.8 & 0.9 & 1.0 \\
\hline$\rho=1.0$ & $\mathbb{D}$ & $\mathbb{D}$ & $\mathbb{D}$ & $\mathbb{D}$ & $\mathbb{D}$ & $\mathbb{D}$ & $\mathbb{D}$ & $\mathbb{D}$ & $\mathbb{D}$ & $\mathbb{D}$ & $\mathbb{D}$ \\
\hline$\rho=0.7$ & $\overline{\mathbb{D}}$ & $\mathbb{D}$ & $\mathbb{D}$ & $\mathbb{D}$ & $\mathbb{D}$ & $\mathrm{C}$ & $\mathrm{C}$ & $\mathrm{C}$ & $\mathrm{C}$ & $\mathrm{C}$ & $\mathrm{C}$ \\
\hline$\rho=0.3$ & $\mathbb{D}$ & $\mathbb{D}$ & $\mathrm{C}$ & $\mathrm{C}$ & $\mathrm{C}$ & $\mathrm{C}$ & $\mathrm{C}$ & $\mathbf{C}$ & $\mathrm{C}$ & $\mathrm{C}$ & $\mathrm{C}$ \\
\hline$\rho=0.0$ & $\overline{\mathbb{D}}$ & $\mathbb{D}$ & $\mathrm{C}$ & $\mathrm{C}$ & $\mathrm{C}$ & $\mathbf{C}$ & $\mathrm{C}$ & $\mathrm{C}$ & $\mathrm{C}$ & $\mathrm{C}$ & $\mathrm{C}$ \\
\hline
\end{tabular}




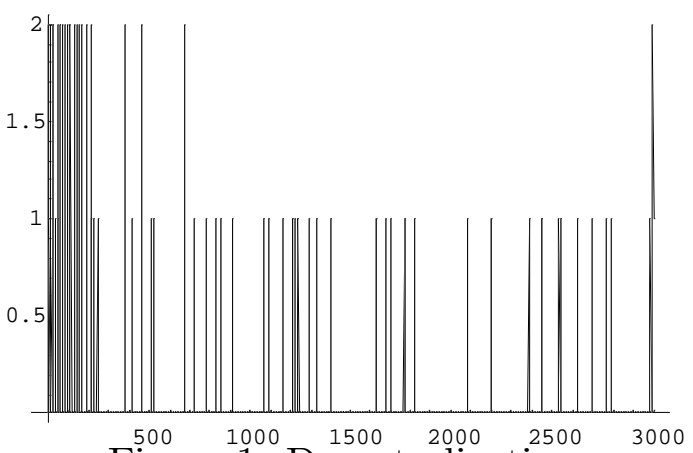

Figure 1: Decentralization

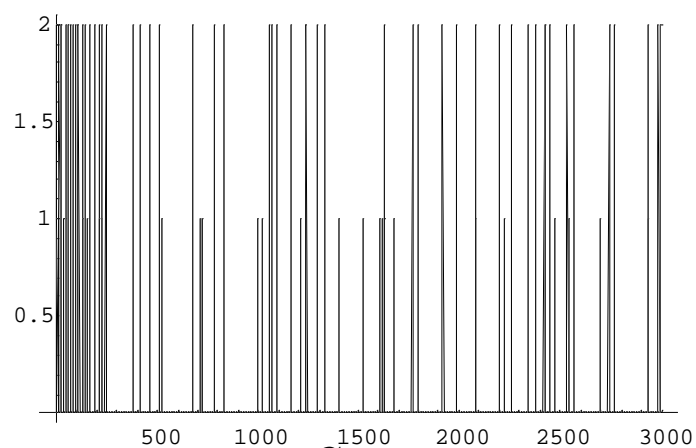

Figure 2: Centralization

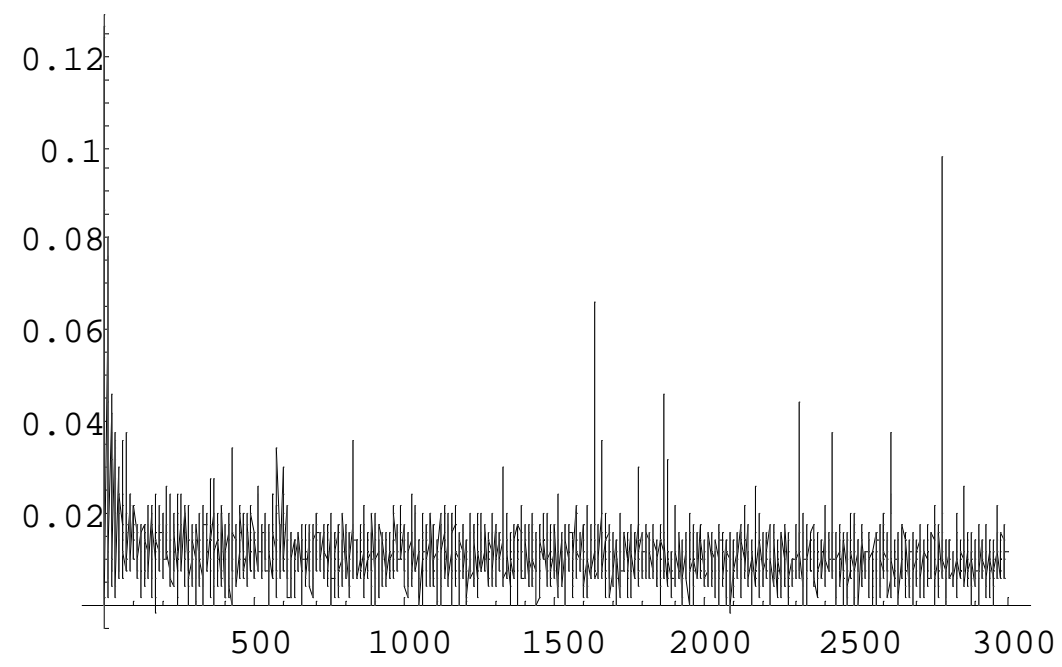

Figure 3: Differential likelihood of a given idea benefitting both stores under centralization and decentralization $[\alpha=1, \gamma=3]$ 


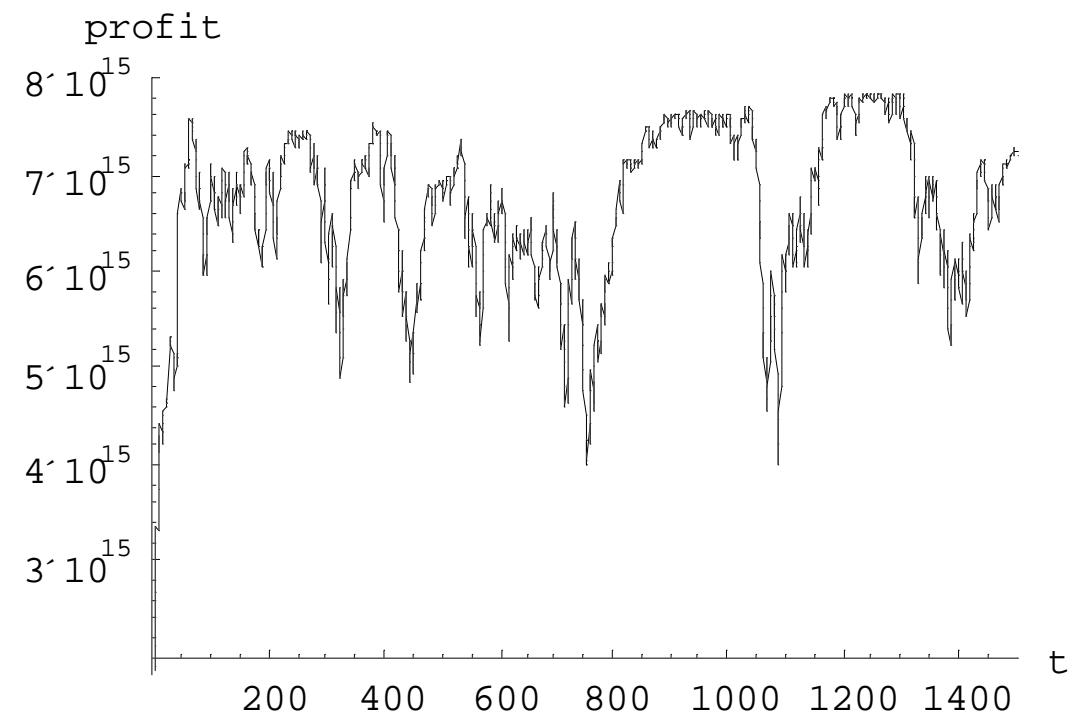

Figure 4: Chain Profit Path over 1500 periods with $\rho=1.0$ and $d=1.0$

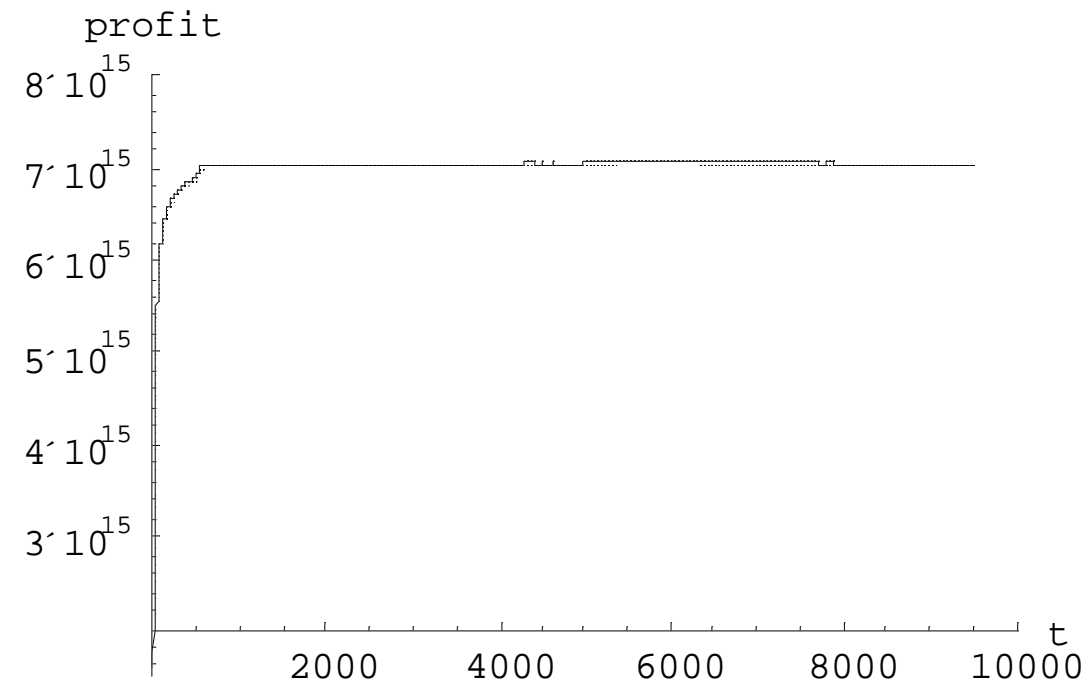

Figure 5: Moving Average Profit Path with $\rho=1.0$ and $d=1.0$ 